\title{
Optical ordering of nanoparticles trapped by Laguerre-Gaussian laser modes
}

\author{
David S. Bradshaw and David L. Andrews* \\ Nanostructures and Photomolecular Systems, School of Chemical Sciences, \\ University of East Anglia, Norwich NR4 7TJ, U.K.
}

\begin{abstract}
In earlier work, it has been established that laser-induced coupling between a pair of nanoparticles can enable the generation of novel patterns, entirely determined and controlled by the frequency, intensity, and polarization of the optical input. In this paper, the detailed spatial disposition about the beam axis is determined for two-, three- and fournanoparticle systems irradiated by a Laguerre-Gaussian (LG) laser mode. The range-dependent laser-induced energy shift is identified by the employment of a quantum electrodynamical description, calculations are performed to determine the distribution of absolute minima as a function of the topological charge, and the results are graphically displayed. This analysis illustrates a number of interesting features, including the fact that on increasing the LG beam's topological charge the particles increasingly cluster, i.e. the order of the structure is significantly raised - also the number of minima for which the particles can be trapped is enhanced. Finally, it is shown that similar principles apply to other kinds of radially structured optical modes.
\end{abstract}

Keywords: Optical vortices, twisted beams, Laguerre-Gaussian beams, quantum electrodynamics, nanoparticles, optical tweezers, stimulated scattering

\section{INTRODUCTION}

Recently there has been a heightened interest in the implementation of 'twisted' Laguerre-Gaussian (LG) modes in optical tweezer instrumentation, in a development that has become known as 'optical spanners'. ${ }^{1}$ In this technique particles are not only trapped but also rotated as a result of angular momentum transfer from the beam., ${ }^{2,3}$ As well as having a rotational influence, such beams have transverse structures that offer novel possibilities for particle trapping. Whilst there is a considerable body of work on the forms of optomechanical coupling involved in optical tweezers and spanners, in which incoming laser light exerts forces on individual particles, it is less recognized that there exist entirely distinct optically induced inter-particle interactions. ${ }^{4,5}$ Last year, by employing a quantum electrodynamical description, the first detailed research was conducted on the laser-induced force between a pair of particles. ${ }^{6-9}$ The latest developments reported here explore the associated energy-shift - directly illustrating the extent and form of particle ordering due to an input LG beam. The significance of the twisted structure is considered through a comparison to the effect of conventional Hermite-Gaussian beams, and the effect of increasing the topological charge, $l$, is also investigated. As well as pairs of nanoparticles, possible extensions to three- and four- particle systems are considered.

\section{LASER-INDUCED INTERACTIONS}

The laser-induced interaction involves the annihilation of a 'real' laser input photon at one particle and the stimulated emission of an equivalent 'real' photon at the other, with a virtual photon acting as mediator between the two particles. This mechanism is represented graphically by two state-sequence diagrams - one of which is shown in figure 1 - or, equivalently, by 48 time-ordered diagrams (two are given by figures $2(a),(b))$. In this work, a pair of nanoparticles $(A$ 


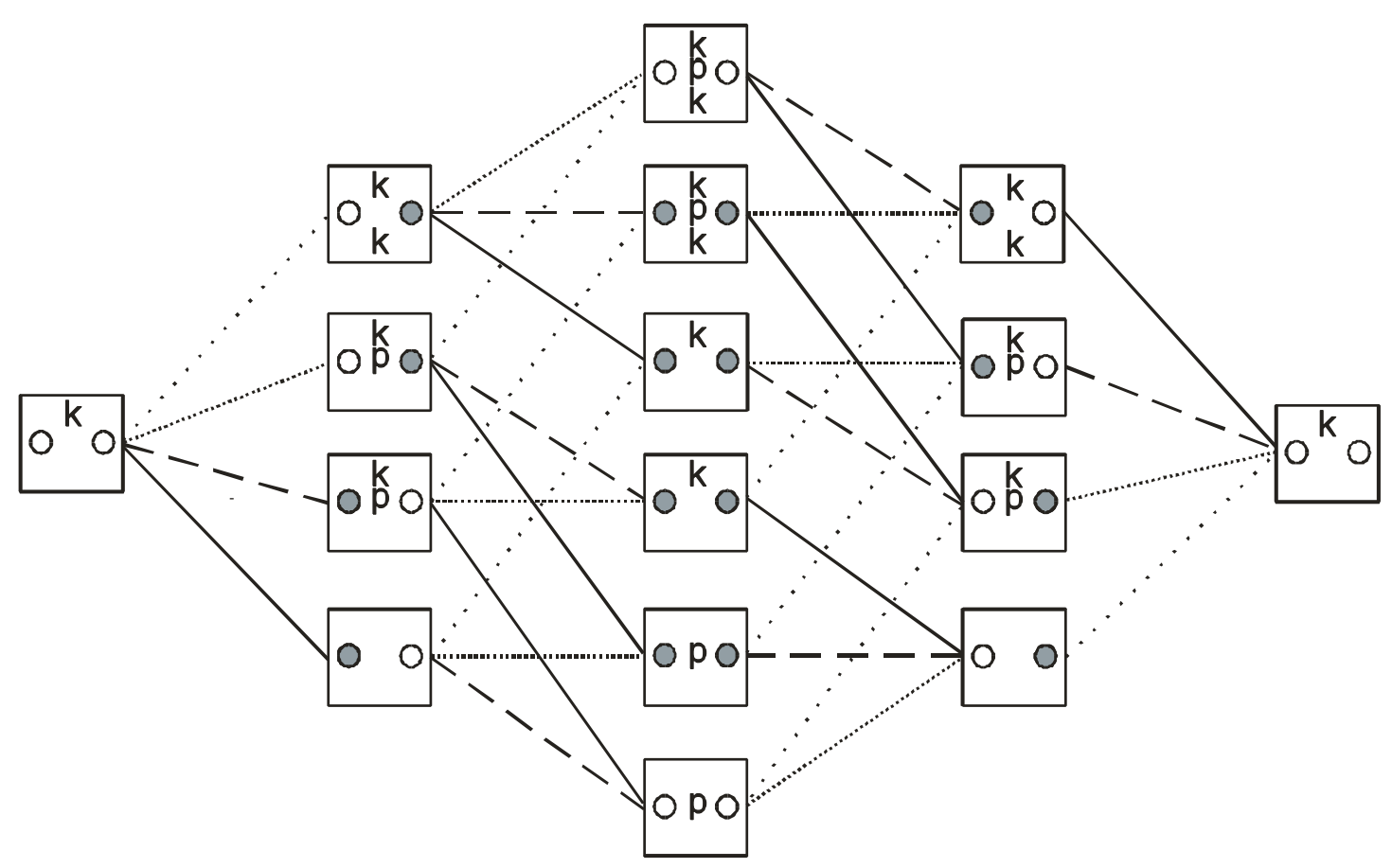

Figure 1. One of two state-sequence diagrams for calculation of the dynamic contributions to the laser-induced interaction, associated with the annihilation of an input laser photon at $A$ and creation of an output laser photon at $B$. Key: $\mathrm{k}$ - 'real' photon, $\mathrm{p}$ - virtual photon, $\mathrm{O}$ - particle in ground state (particle $A$ is a circle on the left-side of the box and $B$ is on the right), $\mathrm{O}$-particle in virtual excited state, - 'real' photon annihilation at $A$,

, - virtual photon coupling event at $A$, ........... - virtual photon coupling at $B$ and, - . - . - - 'real' photon creation at $B$.

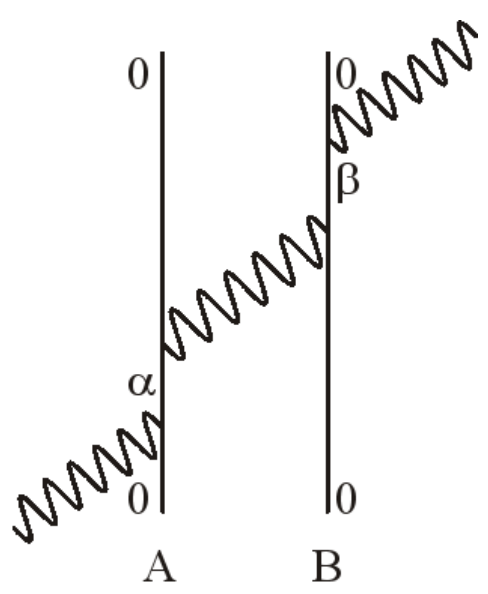

(a)

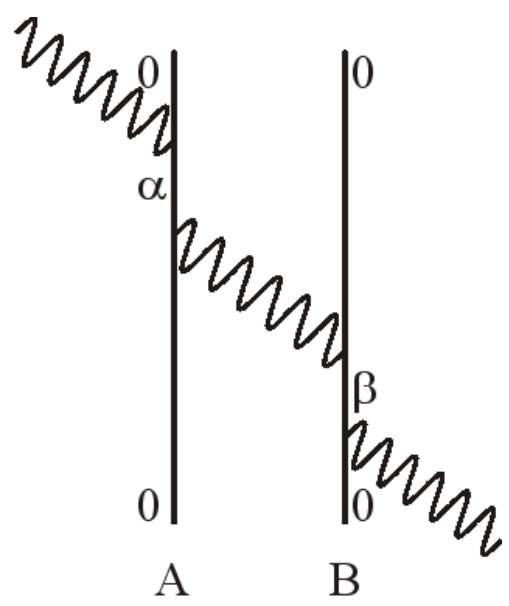

(b)

Figure 2. Typical Feynman diagrams (each with twenty-three further time-ordered permutations) for calculation of the laser-induced interaction energy shift due to stimulated scattering by the pair: 0 denotes the ground state level, $\alpha$ and $\beta$ are excited levels for particles $A$ and $B$, respectively. 


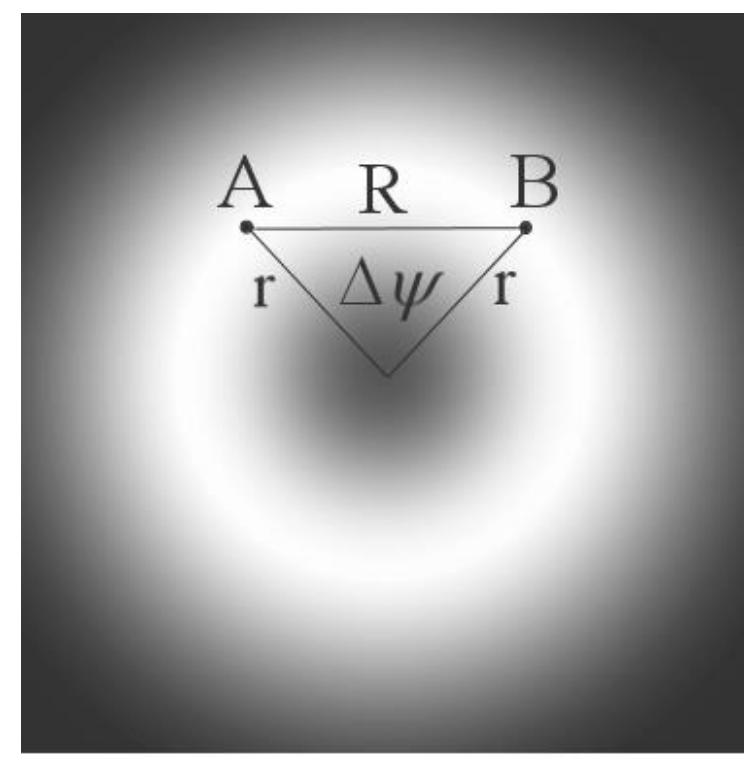

Figure 3. Geometry of a particle pair in a Laguerre-Gaussian beam. Here, $l=1$ and $p=0$.

and $B$ ) are chosen to be optically trapped in an LG beam with arbitrary $l$ and $p=0$, i.e. an optical vortex with one radial node at the beam centre; see figure 3. Further, both particles are equidistant from the beam centre in an optical trap formed by the high-intensity regions, contained within the same cross-section of the beam. Under these conditions, from fourth-order perturbation theory it emerges that the laser-induced energy-shift between the particle pair is given by the expression; 8,9

$$
\Delta E_{A B}=\left(\frac{I f_{l p}^{2} \alpha^{2}}{4 \pi \varepsilon_{0}^{2} c A_{l p}}\right)\left\{\cos ^{2} \phi\left(\frac{\cos k R}{R^{3}}+\frac{k \sin k R}{R^{2}}-\frac{k^{2} \cos k R}{R}\right)-2 \sin ^{2} \phi\left(\frac{\cos k R}{R^{3}}+\frac{k \sin k R}{R^{2}}\right)\right\} \cos (l \Delta \psi) .
$$

where $f_{l p}$ and $A_{l p}$ are beam constants defined in ref. 10, $\alpha$ is the polarisability of a spherical nanoparticle (the same for $A$ and $B$ ), $\hbar c k$ denotes the input photon energy, $\phi$ is the angle between the polarization of the input radiation and $\mathbf{R}$ (the vector displacement between the nanoparticles, whose magnitude is the distance $R$ ), $I$ represents the irradiance and $\Delta \psi=\psi_{B}-\psi_{A}$ is the azimuthal displacement angle. In the short-range region $(k R<<1)$, the leading term of equation (1) is determined from Taylor series expansions of $\sin (k R)$ and $\cos (k R)$. As a result $\Delta E_{A B}$ becomes, using the relation $R=\sqrt{2} r(1-\cos \Delta \psi)^{1 / 2}$;

$$
\Delta E_{A B}^{0}=\left[\frac{I f_{l p}^{2} \alpha^{2}\left(1-3 \sin ^{2} \phi\right)}{8 \sqrt{2} \pi \varepsilon_{0}^{2} r^{3} c A_{l p}}\right] \frac{\cos (l \Delta \psi)}{(\eta-\cos \Delta \psi)^{3 / 2}} .
$$

Here, $\eta$ is a damping factor whose introduction, in place of the unity that emerges from simple trigonometry, prevents equation (2) rising non-physically to infinity as $\Delta \psi$ approaches $0^{\circ}$ (the latter being a feature that would signify overlap of the particles). Treating the term inside the square brackets of equation (2) as constant, for the purpose of this work, 


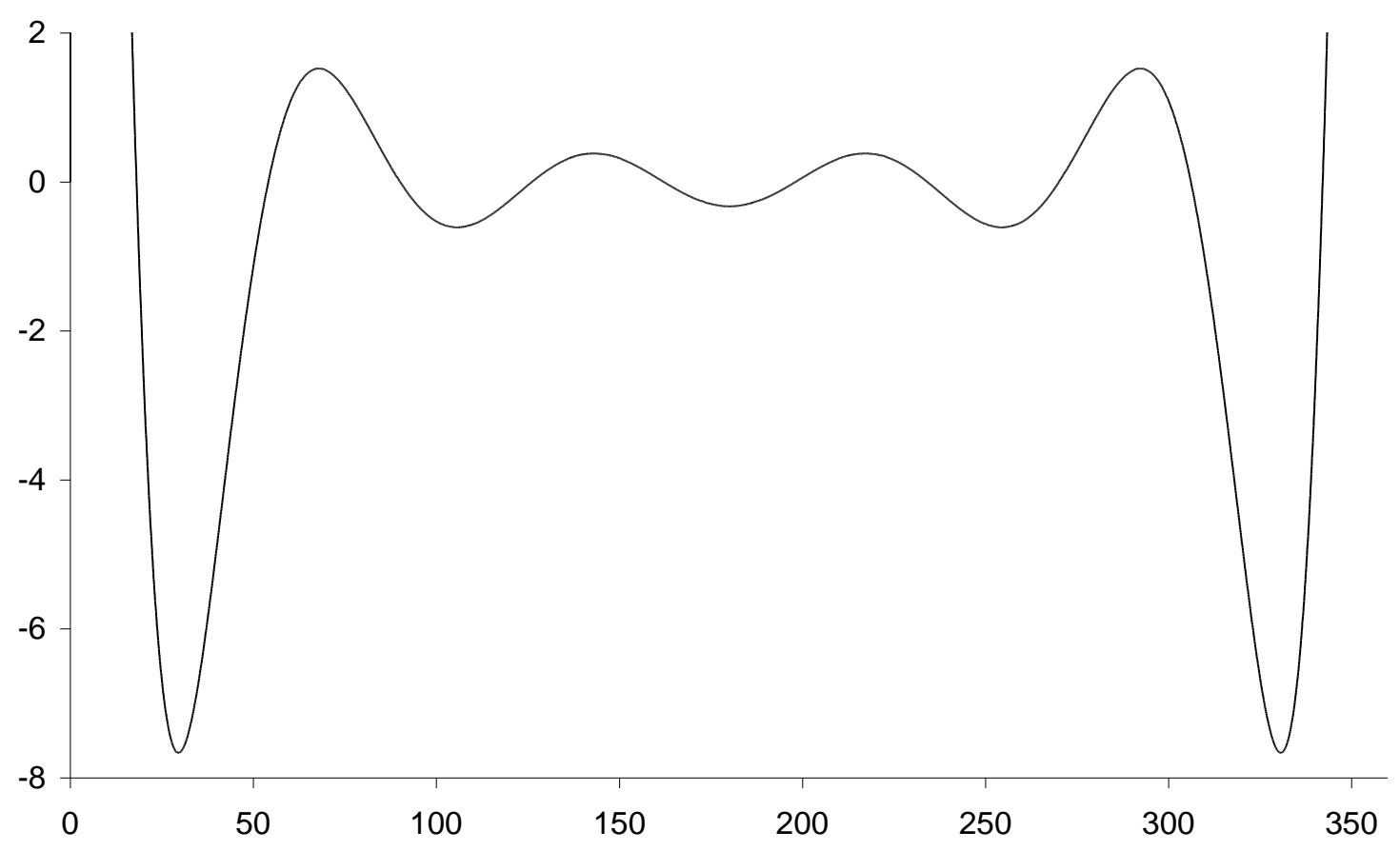

(a)

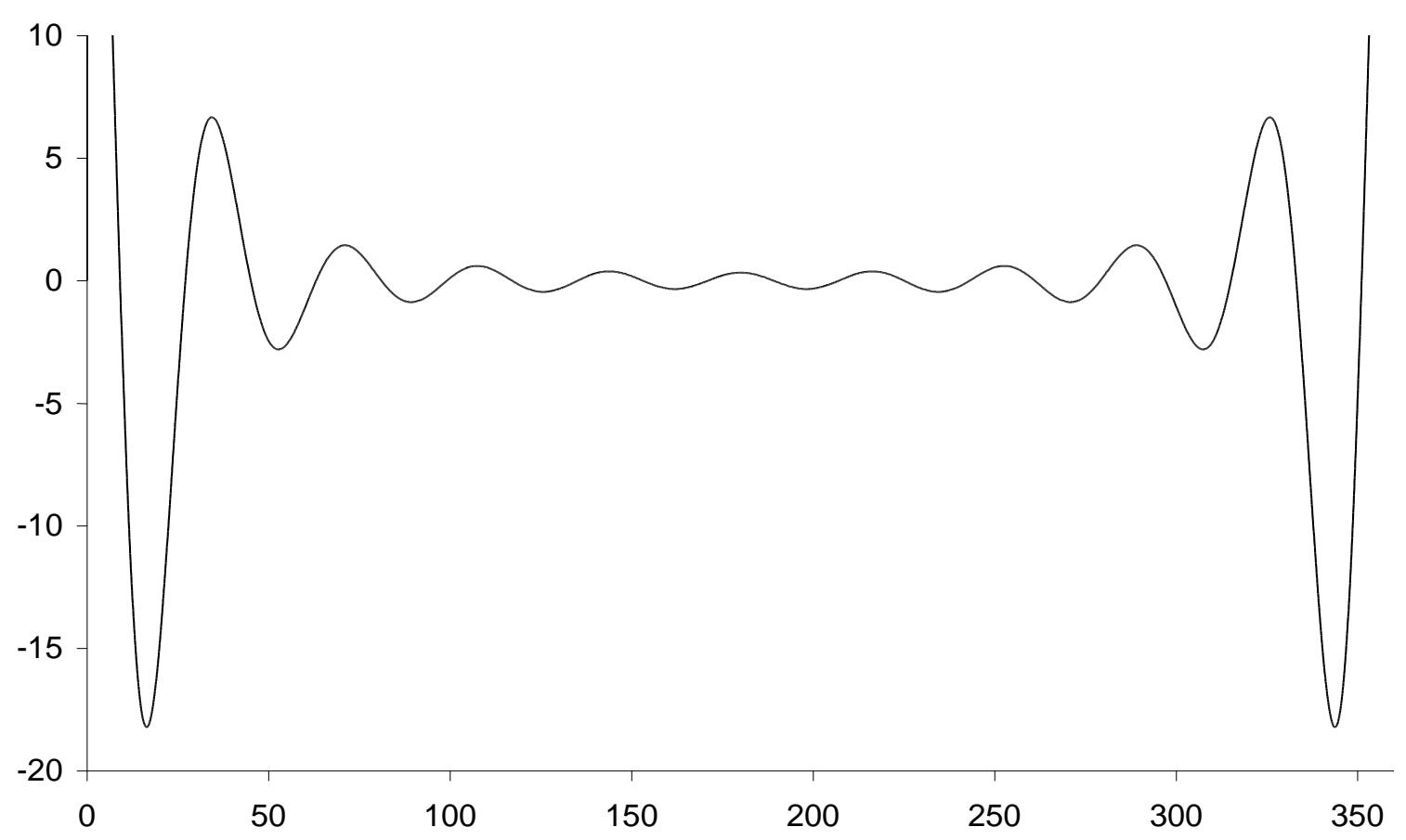

(b)

Figure 4. Plot of $\Delta E_{A B}^{0}$ against $\Delta \psi$ (degrees) for a molecular pair in an LG beam with: $(a) l=5$ and, $(b) l=10$. Here and in the following figures, $\eta=1.1$ is employed in the calculations. 


\begin{tabular}{|c|ccccc|}
\hline$l$ & 0 & 2 & 4 & 10 & 20 \\
\hline$\Delta \psi$ & 180 & 65.2 & 31.8 & 12.6 & 6.3 \\
\hline
\end{tabular}

Table 1. Absolute minima (degrees) of $\Delta E_{A B}^{0}$ for differing $l$.

graphs of $\Delta E_{A B}^{0}$ against $\Delta \psi$ can be constructed for given values of $l$, as illustrated in figures $4(a),(b)$. On detailed inspection of such graphs with varying $l$ it can be verified that: (i) at $l=0$ - i.e. for a conventional Gaussian laser beam the sole minimum is seen at $180^{\circ}$, illustrating that the energetically most favorable position of the particles in the beam cross-section is where they are diametrically opposite each other, as might be expected; (ii) for odd values of $l$ greater than 1, only a local minimum (not the energetically most favorable) arises for this configuration; (iii) for even values of $l$, a local maximum occurs at $180^{\circ}$; (iv) generally, for $l \neq 0$, there are $l$ angular minima and $(l-1)$ maxima. Additional features reflect the behavior associated with increasing values of $l$ : (v) the number of positions for which the particle pair can be mutually trapped increases, becoming less energetically favorable as the angular disposition increases towards diametric opposition, and; (vi) absolute minima are found at decreasing values of $\Delta \psi$ (table 1), physically signifying a progression towards particle clustering. Note, the data in table 1 (and those following) are determined with $\eta=1$, i.e. no damping, as the results are sufficiently remote from values denoting particle overlap - the results are still within a few degrees of those produced by the graphs with $\eta=1.1$ damping accommodated.

The two-particle analysis based on the configuration shown in fig. 2 is easily extended to a three-particle arrangement. In this case $\triangle E_{A B C}^{0}$ is determined by summing the pairwise laser-induced interactions of the three particles with each other, employing variables $\Delta \psi_{1}$ and $\Delta \psi_{2}$ as the azimuthal displacements between particles $A-B$ and $B$ - $C$ respectively. Here, $\Delta \psi_{1}$ and $\Delta \psi_{2}$ are in the interval $\left(0,180^{\circ}\right)$ and the corresponding angular disposition between $A-C$ is dependent on $\Delta \psi_{1}+\Delta \psi_{2}$ unless this exceeds $180^{\circ}$, in which case the dependence is $360^{\circ}-\left(\Delta \psi_{1}+\Delta \psi_{2}\right)$. Note, the term inside the square brackets of equation (2) gives the same values for the interaction of particles $A-B, B-C$ and $A-C$, except for its dependence on $\phi$; although this varies for the differing directions of the inter-particle displacement vector, the variation is slight and for simplicity it is not accounted for. Contour plots of $\triangle E_{A B C}^{0}$ against $\Delta \psi_{1}$ and $\Delta \psi_{2}$ are exhibited in figures 5(a)-(d). Calculations of the absolute minima of $\Delta E_{A B C}^{0}$ with $l=0$ attain the expected values of $120^{\circ}$ for both angles (equidistant particles). For higher values of $l$, as earlier, clustering is observed; for example $l=4$ gives the values $\Delta \psi_{1}=\Delta \psi_{2} \sim 30^{\circ}$.

Extending the analysis to a four-particle system, the corresponding energy-shift $\triangle E_{A B C D}^{0}$ is determined using a similar procedure to that outlined above with the additional variable $\Delta \psi_{3}$, the angle between particles $C$ and $D$. Determination of the minima of $\Delta E_{A B C D}^{0}$ with $l=0$ confirms equidistant values of $90^{\circ}$ for each pair displacement angle; here the value $l=4$ gives clustering with $\Delta \psi_{1} \sim 30^{\circ}, \Delta \psi_{2} \sim 29^{\circ}$ and $\Delta \psi_{3} \sim 30^{\circ}-$ see fig. 6 . Physically, this demonstrates that the inner particle pair $(B$ and $C$ ) clusters to a greater extent than the other pairs due to the additional 

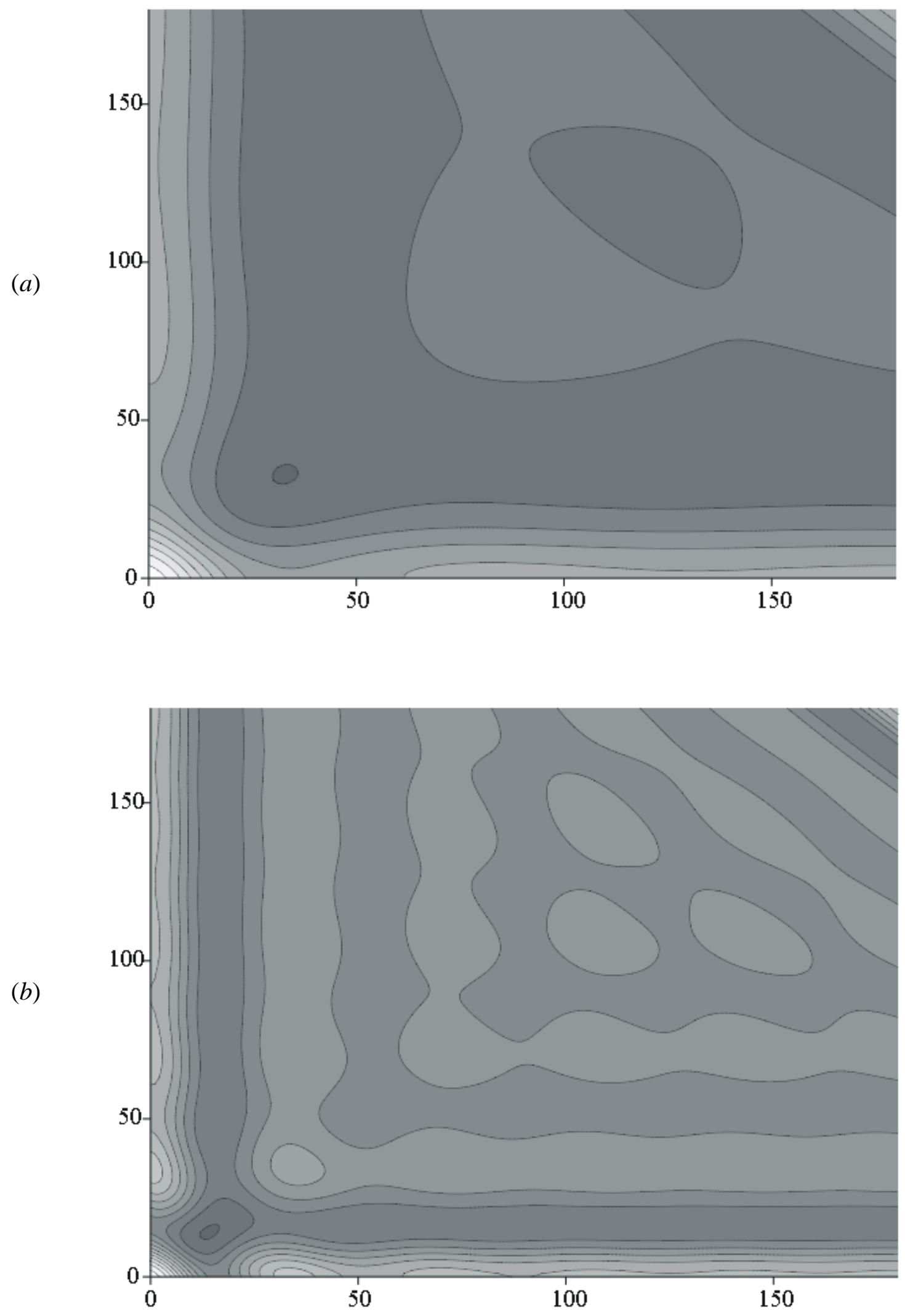

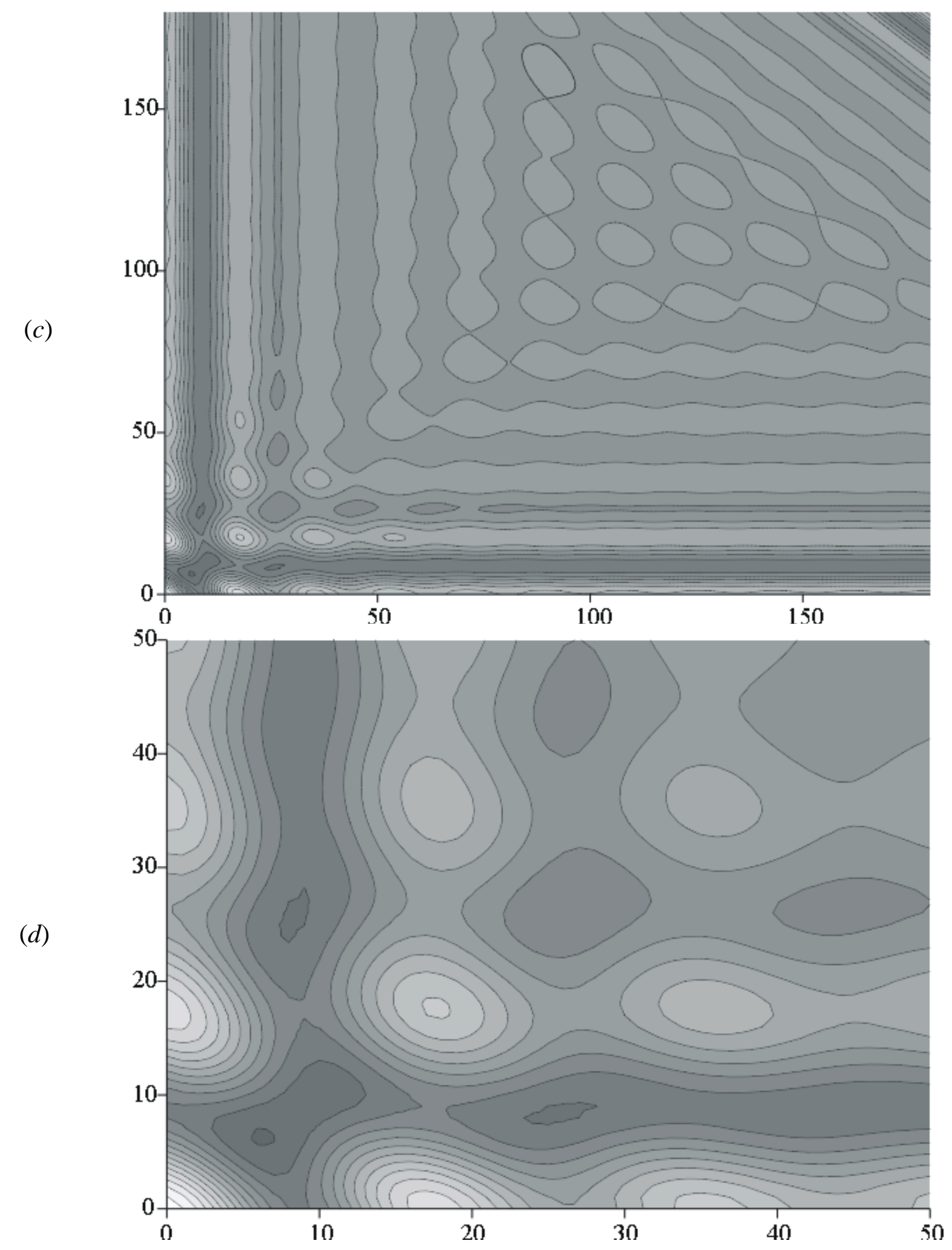

Figure 5. Contour graphs of $\Delta E_{A B C}^{0}$ against $\Delta \psi_{1}$ (x-axis) and $\Delta \psi_{2}$ (y-axis) for three molecules in an LG beam with: (a) $l=4,(b) l=10,(c) l=20$ and, $(d) l=20$ (close-up). Here, lighter shading denotes higher values of $\Delta E_{A B C}^{0}$. 


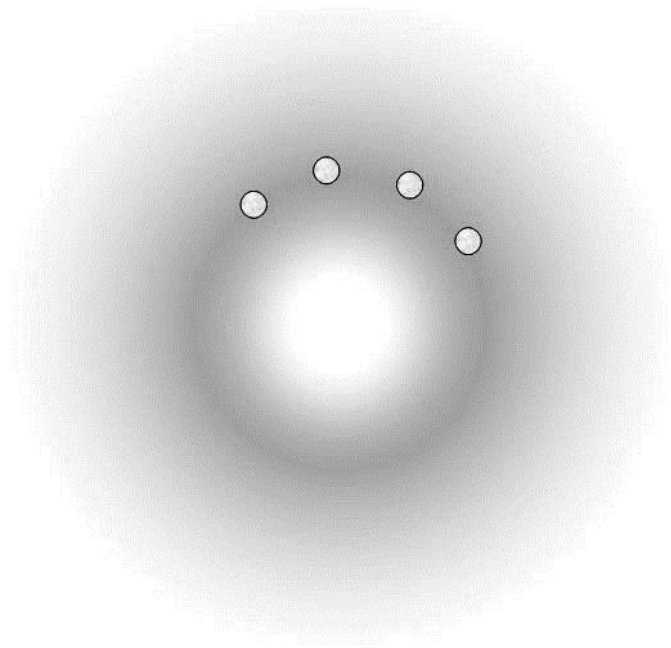

Figure 6. Clustering of nanoparticles in an $l=4$ Laguerre-Gaussian beam

influence of the outer particles $(A$ and $D)$. Further analysis of the three- and four- particle systems for varying values of $l$ reinforces the above features in that: (i) $\Delta \psi_{1}, \Delta \psi_{2}$ for the three-particle case and $\Delta \psi_{1}, \Delta \psi_{3}$ for four particles give similar results - for example, $\sim 60^{\circ}$ for $l=2$ and $\sim 12^{\circ}$ for $l=10$; (ii) in comparison $\Delta \psi_{2}$ is of smaller value for the physical reasons given above - for example, $\sim 56^{\circ}$ for $l=2$ and $\sim 11^{\circ}$ for $l=10$; (iii) there is greater particle clustering for increasing $l$. It may be observed that three-body coupling between the particles is omitted from consideration in the above analysis. However, the complex analytical extension required for its inclusion ${ }^{11,12}$ is not justified since it involves higher order perturbation theory and generates only insignificant corrections.

\section{DISCUSSION}

Although the given results have exemplified cases where $p=0$, the methods employed will also apply directly to cases with other values of $p$, signifying one of the other beam topologies permissible for a given topological charge (the index $p$ determines $(p+1)$ ring-shaped intensity maxima). Experimentally, however, there would be a technical problem in the possibility of particles being optically trapped in one of the other annular intensity regions of the beam. LG modes with $p>0$ are in a sense similar in nature to higher-order Bessel modes, a type of beam also currently employed in optical tweezer systems. ${ }^{13}$ By extension, theory can also be formulated for such beams - except that $f_{l p}$ in equations (1) and (2) are substituted for an $l^{\text {th }}$ order Bessel function - but the same technical difficulty nonetheless arises if two or more annular regions are populated with nanoparticles. Of further interest are other novel light beam structures endowed with orbital angular momentum that could also be applied to optically order nanoparticles. ${ }^{14,15}$ For both LG and higher-order Bessel beams, it is of additional interest to note recent studies on optical vortex production with non-integer $l .{ }^{16}$ In connection with optically-induced inter-particle forces, this is a ground for future exploration.

To conclude, we emphasize that the above findings illustrate the operation of laser-induced mechanisms that are significantly different from those of established 'optical spanner' methods - not least because, here, there is no orbital 
angular momentum transfer from the laser beam. Distinctions can also be drawn against the self-trapping structures of superficially similar appearance that are exhibited by the so-called 'necklace-ring' beams, mathematically equivalent to superpositions of twisted beams with equal but opposite topological charge, which can be formed in self-focusing Kerr media. ${ }^{17-19}$ In the trapping mechanism described above, the light configuration is stable without a supporting medium and there is no need to accommodate complex beam propagation dynamics. The bead structures exhibited by necklacering beams signify localized regions of high intensity, but they are not amenable to particle trapping, primarily because of their necessary spatial localization in the condensed phase. Lastly we note that, assuming the nanoparticles are transparent to the throughput laser radiation, our scheme does not suffer the limitations associated with trapped particles circulating around the annular high intensity region, as in some holographic optical tweezers schemes. ${ }^{20}$ Given the clear advantages of the mechanism we have described, and the relatively straightforward optical setup required, it is hoped that future work by experimental groups will identify the ordering and clustering features identified in this paper.

\section{ACKNOWLEDGEMENT}

The authors gladly acknowledge funding of this work by the UK Engineering and Physical Sciences Research Council.

\section{REFERENCES}

1. M. J. Padgett and L. Allen, “Optical tweezers and spanners”, Physics World 10, pp. 35-38 (1997).

2. N. B. Simpson, K. Dholakia, L. Allen and M. J. Padgett, "Mechanical equivalence of spin and orbital angular momentum of light: an optical spanner”, Opt. Lett. 22, pp. 52-54, 1997.

3. T. A. Nieminen, N. R. Heckenberg and H. Rubinsztein-Dunlop, "Optical measurement of microscopic torque", J. Mod. Opt. 48, pp. 405-413, 2001.

4. T. Thirunamachandran, "Intermolecular interactions in the presence of an intense radiation field", Mol. Phys. 40, pp. 393-399, 1980.

5. M. M. Burns, J.-M. Fournier and J. A. Golovchenko, “Optical binding”, Phys. Rev. Lett. 63, pp. 1233-1236, 1989.

6. D. L. Andrews and D. S. Bradshaw, "Laser-induced forces between carbon nanotubes", Opt. Lett. 30, pp. 783-785, 2005.

7. D. S. Bradshaw and D. L. Andrews, "Optical forces between dielectric nanoparticles in an optical vortex", in Nanomanipulation with Light, ed. D. L. Andrews, Proc. SPIE 5736, pp. 87-95, 2005.

8. D. S. Bradshaw and D. L. Andrews, "Nanoparticle manipulation through inter-particle optical forces and torques", in Optical Trapping and Optical Micromanipulation II, ed. K. Dholakia and G.C. Spalding, Proc. SPIE 5930, pp. 583-591, 2005.

9. D. S. Bradshaw and D. L. Andrews, "Optically induced forces and torques: Interactions between nanoparticles in a laser beam", Phys. Rev. A 72, 033816, 2005.

10. L. C. Dávila Romero, D. L. Andrews and M. Babiker, “A quantum electrodynamics framework for the nonlinear optics of twisted beams”, J. Opt. B: Quantum Semiclass. Opt. 4, pp. S66-S72, 2002.

11. D. P. Craig and T. Thirunamachandran, "Third-body mediation of resonance coupling between identical molecules", Chem. Phys. 135, pp. 37-48, 1989. 
12. R. Passante, E. A. Power and T. Thirunamachandran, "Radiation-molecule coupling using dynamic polarizabilities: Application to many-body forces”, Phys. Lett. A 249, pp. 77-82, 1998.

13. D. McGloin and K. Dholakia, "Bessel beams: diffraction in a new light", Contemp. Phys. 46, pp. 15-28, 2005.

14. S. Chávez-Cerda, M. J. Padgett, I. Allison, G. H. C. New, J. C. Gutiérrez-Vega, A. T. O’Neil, I. MacVicar and J. Courtial, "Holographic generation and orbital angular momentum of higher-order Mathieu beams", J. Opt. B: Quantum Semiclass. Opt. 4, pp. S52-S57, 2002.

15. E. J. Galvez, P. R. Crawford, H. I. Sztul, M. J. Pysher, P. J. Haglin and R. E. Williams, “Geometric phase associated with mode transformations of optical beams bearing orbital angular momentum”, Phys. Rev. Lett. 90, 203901, 2003.

16. J. Leach, E. Yao and M. J. Padgett, "Observation of the vortex structure of a non-integer vortex beam”, New J. Phys. 6, 71, 2004.

17. M. Soljačić, S. Sears and M. Segev, "Self-trapping of 'necklace' beams in self-focusing Kerr media”, Phys. Rev. Lett. 81, pp. 4851-4854, 1998.

18. M. Soljačić and M. Segev, "Self-trapping of 'necklace-ring' beams in self-focusing Kerr media”, Phys. Rev. E 62, pp. 2810-2820, 2000.

19. M. Soljačić and M. Segev, "Integer and fractional angular momentum borne on self-trapped necklace-ring beams", Phys. Rev. Lett. 86, pp. 420-423, 2001.

20. J. E. Curtis, B. A. Koss and D. G. Grier, "Dynamic holographic tweezers”, Opt. Comm. 207, pp. 169-175, 2002.

*david.andrews@physics.org 